\title{
GPU BASED TOOLBOX FOR FUZZY LOGIC SYSTEM USING WHALE OPTIMIZATION ALGORITHM
}

\author{
Sarabjeet Singh \\ Department of Computer Science \& Engineering (Degree Wing), \\ Chandigarh College of Engineering and Technology, Sec-26, Chandigarh, India

\section{Dr. Satvir Singh} \\ Electronics \& Communication Engineering, \\ I.K. Gujral Punjab Technical University, Kapurthala, Punjab, India

\section{Dr. Vijay Kumar Banga} \\ Amritsar College of Engineering and Technology, Amritsar, India
}

\begin{abstract}
Fuzzy Logic System (FLS) is an efficient method to solve engineering problems. However, the training of a Fuzzy Logic System is a time-consuming task. Optimization Algorithm can be used to optimize the rule base of any FLS. Out of Type-1 FLS and Type-2 FLS, the type-2 found to be more effective to deal with noisy data. Due to their computational requirements Interval Type-2 (IT2) has been preferred over General Type-2. Whale Optimization Algorithm (WOA)has been introduced recently. The algorithm has been tested on different engineering problems and is found to be more effective. General Purpose Computing using graphics Processing Unit (GPGPU) is a new way to solve compute intensive problems on Graphics Processing Unit (GPU). CUDA-C is a parallel language that can be used to execute parallel code NVIDIA GPU. This paper integrates IT2 FLS, WOA and Processing Power of GPU. A toolbox is proposed that can be used to optimize the rule base in parallel. The toolbox provides both the implementations, i.e. serial and Parallel. FLS WOA Toolbox is design in such a way that user can pass parameter dynamically according to their need without interfacing with the code.
\end{abstract}

Key words: Whale Optimization Algorithm, CUDA-C, Interval Type-2 Fuzzy Logic System, Graphics Processing Unit.

Cite this Article: Sarabjeet Singh, Satvir Singh and Vijay Kumar Banga, GPU Based Toolbox for Fuzzy Logic System using Whale Optimization Algorithm International Journal of Computer Engineering and Technology, 10(1), 2019, pp. 11-24.

http://iaeme.com/Home/issue/IJCET?Volume=10\&Issue $=1$ 


\section{INTRODUCTION}

Artificial Intelligence can be used to solve engineering problems effectively. Optimization is an AI based technique that is used to imitate natural behaviour of living organisms. These algorithms model the behaviour mathematically and it is found that algorithms can solve any class of problems more precisely and in less time. Whale optimization Algorithm is a recently developed algorithm that has been tested on different engineering problems successfully [1]

Fuzzy Logic System, generally referred as Type-1 (T1) FLS was introduced by Lofti A. Zadeh [2]. FLS has been used by researchers to solve different problems in a precise manner. General Type-2(T2) FLS has been found to be more accurate than T1 FLS [3] as it can handle noisy signals more accurately. However, due to their computational complexity Interval Type2 (IT2) is preferred over General T2 FLS. The computational requirements of IT2 is less than General T2, but accuracy is acceptable.

During last decade, Graphic Processing Unit (GPU) has been used widely as a powerful parallel processing component. Nowadays, use of GPU has become popular and use of the GPU for general purpose computing termed as General Purpose Computing on Graphic Processing Unit (GPGPU)[4][5]. As compared to CPU GPU is fast computational device because of its underlying stream processing architecture. GPU is much more economical as compared to CPU. There has been tremendous research for development of platforms that supports general purpose computing on GPU such as OpenGL by Silicon Graphics, Brook GPU (Stanford University) [6]. NVIDIA Corporation GPUs supports CUDA-C [7]. NVIDIA has advanced standard C language further by adding set of keywords that help in parallel programming. The beauty of CUDA-C is that

it is compatible with Visual Studio. The programmer can interact with GPU by using CUDA-C under Visual Studio Environment and it is easy to use for regular C programmer.

This paper has been divided into ten sections. Section II briefs about optimization algorithms. In section III, Whale Optimization Algorithm has been detailed. Section IV presents Fuzzy Logic System. CUDA Programming Model has been detailed in section V. Section VI briefs about Mackey Glass Time Series. Section VII introduces implementation of serial and parallel on toolbox. Finally, in section VIII and IX results and conclusion has been presented.

\section{OPTIMIZATION ALGORITHMS}

Meta-heuristic optimization algorithms have been considered pervasive arce type solutions for getting an optimum solution from non-linear, multivariable and multimodal functions since last decade due to their easy implementation, no gradient information required, bypass local optima and a wide range of solutions for holding better approximations [1].

Meta-heuristic optimization algorithms provide solution to problems by imitating biotic or some physical concept. As per literature, these may be divided in three types, i.e., evolutionbased [8], physical based [9] or swarm-based [10]. The first approach is evolution based starts by generating a random population. The population is evolved and generates a better solution for the next generation. The GA is the most popular evolution-based optimization algorithm. GA is based on the Darwinian evolution theory [11]. The recent application exists in this category is Biogeography-based optimization (BBO) [12].

The second type is based on a physical concept. These algorithms imitate physical rules existing in the universe. The most popular in these are simulated annealing (SA) [13], GSA [14].

The Third Group mimic the social behavior of social beings. The most popular algorithm is PSO. This algorithm is based on the social behavior of bird's flock. It first randomly assigns the position of all particles. These moving particles move in space and learn from their 
neighbors. Another famous algorithm in this group is Ant Colony Optimization (ACO). In [15], the authors have proposed the grasshopper algorithm and applied to structural design problems for optimization.

This is pertinent to mention that these algorithms are based on social behavior. Although there are many more algorithms that are developed or in process.

Meta-heuristic optimization algorithms that are inspired by nature and based on population, have some a common feature. The search procedure has two phases known as exploration and exploitation [16] [17] [18]. Exploration is the process in which the search space under consideration is initialized using uniformly distributed random values and whole search space is covered. Exploitation search nearby its areas of the solution set. It varies solution minutely and optimizes locally. It helps to search for solutions in nearby areas as changes are made on small scale.

Optimization helps to generate the optimal solutions for the variables of a specific problem by minimizing or maximizing a function based on some objective. Optimization is required in all fields of science [19]. In order to solve any problem using optimization, the following knowledge is required. In phase 1, the parameters of the problem should be determined, in the next phase, the parameter constraints that bound the parameters are to be specified and last the objective of the problem should be identified [1]. In order to design an optimum problem solver, all the above parameters should be considered. Any optimization problem can be solved either mathematically or by using a stochastic method. Due to local optima entrapment problem, i.e., the optimizer gets trapped in local solutions and failure of this model to solve compute-intensive problems.

Stochastic methods are based on the operators that are random in nature and helps to avert local optima entrapment problem [20]. The process starts by creating a set of random solutions for a specific problem. Then a solution is evaluated using an objective function or set of functions. To further improve the solution decisions are taken relying on the values evaluated by the objective function. Thus, algorithms are very effective to solve real-life problems when search space is enormous or unknown.

Nature inspired population-based algorithms are most popular [21]. These algorithms imitate natural problem-solving procedures derived or learned from creatures. These are based on the phenomenon of survival of fittest. To survive, they keep on evolving and adapting using different techniques. Human beings use these techniques for optimizing different problems by observing nature. Nature-inspired algorithms can be based on single solution or can be multisolution [22]. In a single solution based only one random solution is produced and optimized. However, in multi-solution-based algorithms, multiple solutions are produced and improved to find an optimal solution. As per literature, multi-solution-based algorithms are more popular as they are more resistance to local optima [23]. In these algorithms, other solutions help in providing a trapped solution to jump out of local optima. Multi-solution can explore search space more effectively. Solutions exchange information between them for optimization. However, more evaluations are required in these algorithms.

GAs, PSO, ACO [24] and DE [25] are most popular algorithms among multi-solution-based algorithms. Authors have proposed an algorithm based on sine and cosines [26]. The authors proposed prediction of time series using convolution neural network [27].

In GA different operations selection, crossover and mutation help to evolve solutions during each iteration. In the PSO algorithm, the agents imitate the behavior of a flock of birds or school of fish. All solutions are improved as a poor solution and move towards the best solution which is updated after each iteration. In ACO, the concept of the shortest route is used to optimize the solution. DE evolves existing solutions to give the optimal solution. Recently, WOA has been proposed. This Algorithm relies on the killing mechanism of humpback whales. These creatures 
first encircle the prey then they reduce the circle width to hunt the prey. The algorithm has been tested on various optimization problems [1].

A new approach proposes an ANFIS - VOA mechanism, that is integration of ANFIS and a new optimization algorithm known as vortex optimization algorithm (VOA) for time series forecasting [28]. A method as a combination of an autoregressive (AR) model, moving average (MA) model, and T1FF approach into one algorithm for time series forecasting [29]. The rainfall time series had been generated by the seasonal autoregressive integrated moving average (SARIMA) model that predicts a future rainfall the coming years and shown as graph [30]. In [31], authors propose an innovative forecasting model for functional time series which can be used in power system applications.

\section{WHALE OPTIMIZATION ALGORITHM}

Whale is an amazing mammal. Whales are believed to be the biggest mammals on the planet earth. Most of the species of whale are predators. Whales are believed to be the intelligent mammals with emotions. Whales reside in groups. Few whale species (killer whales) nurture as a family for life time. One species of killer whale known as Humpback whale is huge in size. These mammals work in a group for hunting. They use specific killing mechanism. Their foraging character is known as bubble-net feeding method. Whale algorithm works on exploration and exploitation, based on the values of parameters generated. During implementation, the probability value is input in the algorithm and changed after a fixed number of iterations. This variation improves the result of the prediction.

The behavior of exploration and exploitation of whales is expressed mathematically by two approaches.

\subsection{Shrinking Encircling Mechanism}

All whales move toward best search particle. This can be represented by equations.

$$
\begin{aligned}
& \vec{D}=\left|\vec{C} \cdot \vec{X}^{*}(t)-\vec{X}(t)\right| \\
& \vec{X}(t+1)=\bar{X}^{*}(t)-\vec{A} \vec{D}
\end{aligned}
$$

where t indicates the current iteration, $\vec{A}$ and $\vec{C}$ are coefficient vectors. $\overrightarrow{X^{*}}$ denotes position vector of best solution and $\vec{X}$ denotes position vector.

The vectors in Eq.(2) is and Eq.(1) are calculated using Eq.(3) and Eq.(4)

$$
\vec{A}=2 \cdot \vec{a} \cdot \vec{r}-\vec{a}
$$

$\vec{r}$ known as random vector in $[0,1]$. $\overrightarrow{\mathrm{a}}$ lies between 2 to $0 . \overrightarrow{\mathrm{A}}$ and $\overrightarrow{\mathrm{C}}$ are coefficient vectors.

$$
\vec{C}=2 \vec{r}
$$

where as in Eq. (4) $\vec{r}$ is a random number in $[0,1]$.

\subsection{Spiral Updating Position}

This is mathematically expressed by the equation.

$$
\vec{X}(t+1)=\vec{D} \cdot e^{b l} \cos \left(2 \pi l+\vec{X}^{*}(t)\right.
$$

Where as in Eq. (5) $\vec{D}=\left|\overrightarrow{X^{*}}-\vec{X}(t)\right|$ denotes distance between whale and prey. 1 lies between $[-1,1]$, pi is a constant and $\mathrm{b}$ is a constant. 


\subsection{Search for prey}

The search mechanism for prey is modeled by the following equation.

$$
\begin{aligned}
& \vec{D}=\left|\vec{C} \cdot \vec{X}_{\text {rand }}-\vec{X}\right| \\
& \vec{X}(t+1)=\vec{X}_{\text {rand }}-\vec{A} \cdot \vec{D}
\end{aligned}
$$

Algorithm 1 Algorithm for time series forecasting using FLS and WOA

Initialize the whales population $X i(i=1,2, \ldots, n)$

$X^{*}=$ the best search agent

While $(\mathrm{t}<$ max_no_iterations $)$

For each search agent

Update a, A, C, 1, and $\mathrm{p}$

if1 $(\mathrm{p}<0.5)$

if $2(|\mathrm{~A}|<1)$

Update the position of the current search agent Eq. (2)

elseif2 $(|\mathrm{A}|>=1)$

select random search agent (Xrand)

Update the position of the current search agent Eq. (7)

End if2

If $(p>=0.5)$

Update the position of the current search agent Eq. (5)

End ifl

End for

Check if any agent goes beyond the search space amend it

Calculate the RMSE value of FLS of each search agent

Update $X^{*}$ if there is a better solution

$\mathrm{t}=\mathrm{t}+1$

end while

return $X^{*}$

\section{FUZZY LOGIC SYSTEM}

Any type of FLS has ability to solve problems related to data in numbers or lexical data. It is the mapping of input data in the vector form to the output of scalar form. Fuzzy sets used in the FLS for solving any problem. A type-1 Fuzzy set can be used with FLS, however when there is uncertainty in the input signal (noisy) Type-1 fuzzy set is unable to predict the correct output as compared to a Type-2 Fuzzy set. T1 FLS has certain uncertainties associated (1) As words mean distinct to individual people antecedents and consequents that represent the rules may be uncertain (2) Consequences may have the histogram of values associated with them, specifically when information is extracted from the people those disagree. (3) The input data that is used to generate fuzzy sets may be noisy. Type -1 Fuzzy set are crisp value so they are not able to model such systems, however because of the fuzzy nature of Type-2 fuzzy sets they can be used to model such systems. As membership function used for T1 fuzzy sets is of two dimensions and membership functions of T2 fuzzy sets have three dimensions. This additional degree of Type-2 fuzzy sets provides additional knowledge to deal with uncertainties associated with the system. 
There are methods to handle the uncertainty associated with Type-2 fuzzy sets first approach is averaging the responses and the second one is preserving all the responses. The method selected is based on the resources present to deal with a particular problem. In literature averaging of the responses is used because the resource requirement is less as there is no need to preserve all the responses. If one needs more information the second approach is preferred.

\subsection{Type-2 Fuzzy Logic System in Brief}

Zadeh introduced the concept of T2 FSs as an advancement of the notion of T1 FSs in 1975 [2]. T1 FLS can be extended to T2FLS which depicts uncertainty by using an additional dimension [32]. The third dimension of T2 FLS provides further degrees of freedom, as uncertainty can be represented more precisely than T1 FSs. Fuzzy set that ate T2 helps in applications when the evaluation of exact representation function is not easy. When T2 FLS is used the ability to cater a next level of uncertainty raises, it gives those components that are missing and are not shown. If a fuzzy system is deployed carefully it can solve problems where decision making is required.

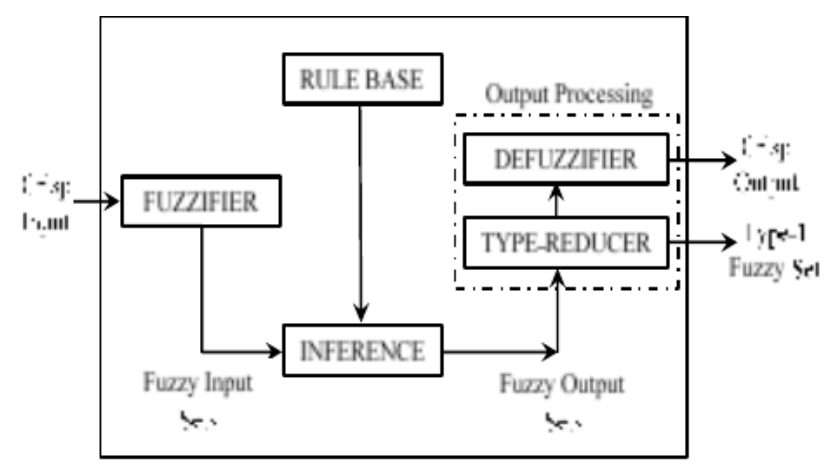

Figure 1 Components of T2 FLS

Any T2 FLS composed of three components along with conversion mechanism that reduces $\mathrm{T} 2$ to $\mathrm{T} 1$ as presented in Fig. 1. An output unit consists of the type-reducer and mechanism that produces a T1 FS output a crisp number. The T2 FLS main characteristic is that it uses T2 FSs for antecedents and/or consequents along with rules represented by IF-THEN.

\subsection{Interval Type-2 Fuzzy Logic System}

General T2 FLSs are more compute intensive than T1 FLS as it contains FSs that has additional dimension. As per research, the concept can be simplified, if secondary membership function is used making them interval sets. These secondary membership values are assigned 0 or 1 and such set has been referred as Interval Type-2 FSs or IT2 FSs in short. As generalized FSs are more compute intensive as than IT2 they have got attention of researchers, as they are easy to use. Due to this more information is present about IT2 FSs than generalized T2 FSs. Researchers nowadays, around the world, are actively investigating both kinds of fuzzy sets.

IT2 FSs has been widely used as they give more degrees of freedom to model higher order of uncertainty as compared to T1 FSs. This feature has been the moving factor behind further improvements in development of new concepts of IT2 FSs and FLSs. IT2 FS is represented by upper and lower boundary of uncertainty known as Upper Membership Function (UMF) and Lower Membership Function (LMF) as shown in Fig.2 The space within upper and lower boundary of Uncertainty is known as Footprint of uncertainty (FOU). 


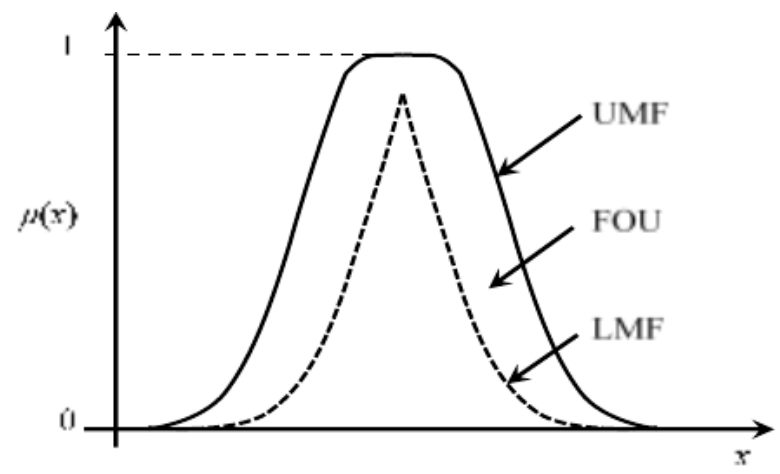

Figure 1 Representation of Interval Type-2 Fuzzy Set.

As stated by Zadeh, a fuzzy set is defined for a particular domain, and is represented with the help of a membership function and is used to map components from the one domain to realvalued numbers [33]. Jerry Mendel along with his students had implemented different techniques to design FLSs for applications of different domains [34]-[37] based on the IT2 FSs as proposed by Zadeh in 1975[2].

In this paper, 4 IT2 FSs have been used to fuzzify the input. Each IT2 FS is framed using two membership functions of type T1 FSs, $\left(\mu_{\mathrm{L}}(\mathrm{x})\right.$ and $\left.\mu_{\mathrm{R}}(\mathrm{x})\right)$ that has same standard variance but distinct means $\left(\mathrm{m}_{\mathrm{L}}\right.$ and $\left.\mathrm{m}_{\mathrm{R}}\right)$. Mathematically, modeled as $(8)$.

$$
\mu_{L}(x)=e^{\left(\frac{-\left(x-m_{L}\right)^{2}}{2 \sigma^{2}}\right)} \text { and } \mu_{R}(x)=e^{\left(\frac{-\left(x-m_{R}\right)^{2}}{2 \sigma^{2}}\right)}
$$

In IT2 FLS, UMF, LMF is evaluated by the union and intersection of set that is on left and set that is on right T1 FSs as depicted in (9). In this, upper and lower bounds of LMF and UMF depicts an uncertainty in an IT2 antecedent FS, A, as presented in Fig.2.

$$
\underline{\mu}_{\mathrm{A}}(\mathrm{x})=\mu_{\mathrm{L}}(\mathrm{x}) \cap \mu_{\mathrm{R}}(\mathrm{x}) \text { and } \mu_{\mathrm{A}}(\mathrm{x})=\mu_{\mathrm{L}}(\mathrm{x}) \cup \mu_{\mathrm{R}}(\mathrm{x})
$$

In this paper, a demonstration of an IT2 FLS with multiple (4) inputs and single output and used for predicting the Mackey-Glass time series [36] [38] using parallel computation. Here Consequents that serve as output of FLS are singleton interval sets, and shown as $\left[\mathrm{C}_{\mathrm{Li} \mathrm{CRi}}\right]$ for $\mathrm{i}=1,2, \ldots$ The implication and aggregation operations are performed using standard min and max standard operators. The method of defuzzification is used, i.e., height defuzzifier. Because the applied FSs are symmetrical, due to this the output of defuzzifier will be equal to as that of the center of gravity technique. As per mathematical model, after firing $P$ rules the aggregated FSs, $B$, is modified by defuzzifier and the crisp output is produced by using (10)-(11)

$$
\begin{aligned}
& y_{L}=\frac{\Sigma_{i=1}^{M} C_{L i} \underline{\mu}_{B}(y)}{\sum_{i=1}^{M} \underline{\mu}_{B}(y)} \\
& y_{U}=\frac{\Sigma_{i=1}^{M} C_{R i} \bar{\mu}_{B}(y)}{\sum_{i=1}^{M} \bar{\mu}_{B}(y)} \\
& y=\frac{1}{2}\left(y_{L}+y_{U}\right)
\end{aligned}
$$

The Scope of parallel computation processing that is possible is described further: In [39] and [40], authors had evaluated T1 FLSs, using 2 inputs and single output, to provide concurrency during firing of multiple fuzzy rules to cater increased sample rate of universe of discourse both for antecedents and consequents. Following same patterns authors in [41] had demonstrated scope of parallelism in a novel way for IT2 FLSs. However, in this paper, a 
specific FLS with 4 antecedents and single consequents had been experimented for execution of multiple rules and by increasing input concurrently.

\section{CUDA PROGRAMMING MODEL}

CUDA developed by Nvidia is a platform for parallel computation and programable paradigm that can be used by any researcher to speed up computational power substantially by utilizing the parallel architecture of the GPU. CUDA provides mechanism so that there can be direct access to the virtual instruction set and memory of the parallel computing tiny processors of GPUs that supports CUDA. A CUDA platform is used to access CUDA-accelerated libraries, compiler directives and extensions to a programming language that are defined as per industrystandards, including $\mathrm{C} / \mathrm{C}++$ and Fortran. With knowledge of $\mathrm{C} / \mathrm{C}++$, one can exploit one or more GPUs on an ordinary desktop computer using CUDA for parallel programming.

CUDA can be used by researchers to run multiple kernels simultaneously at the same time on cores of GPU. CUDA supports a structure named grid and defined as a arrangement of blocks, and one block can run the similar global function using multiple threads, mutually, independent of each other. A thread is the smallest available unit on a GPU as shown in Fig.3.

A block consists of SIMD (Single Instruction Multiple Data) threads that execute at the same time on cores. All threads within a block communicate and synchronize by using shared memory. Each thread executes the same instruction with different sets of data. CUDA programming model programming model gives an interface and allows software developers to divide a user procedure into many small programs, each of which runs concurrently in a different block. Each small program can be again partitioned into small pieces that execute the same set of instructions, however, runs on separate thread concurrently sharing the block. Data set parallelism is achieved by partitioning the data sets into smaller chunks and resides in the shared memory, and each chunk can be accessed only by using threads of that block. Because of this local data placement, the use of global memory is reduced, which minimizes data access time.

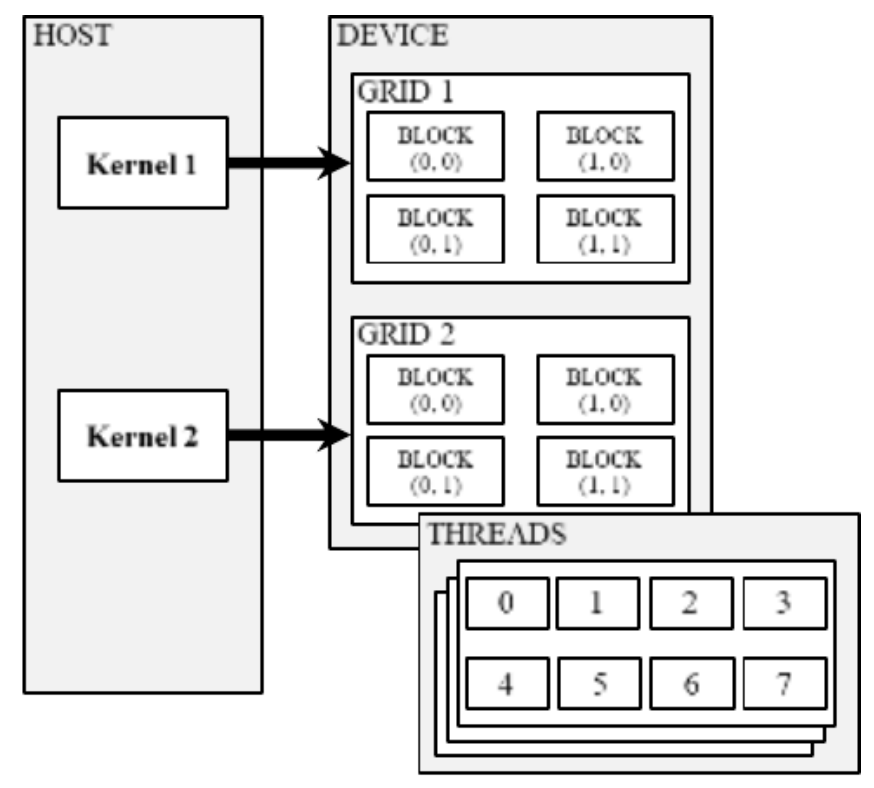

Figure 3 CUDA Programming Architecture

The other feature of the CUDA programming model that is critical is the memory array system as depicted in Fig. 4. To cater maximum applications cuda provides different types of memory and all exhibit its own access time. The memory of GPU can be partitioned into readwrite per-thread registers, read-write per thread-local memory, read-write per-block shared 
memory, read-write per-grid global memory, read-only per-grid constant memory, and readonly per-grid texture memory. As Global memory gives maximum latency a compared to texture and constant memory, so they are more-efficient and they have less access latency time. To use GPUs processing power developers should reduce the number of global memory fetch operations. This can be attained if all threads are allowed to read data using global memory and place the same content into shared memory.

CUDA statement cudaMalloc can be used to allot memory on GPU (device) and using $\mathrm{C} / \mathrm{C}++$ normal malloc keyword on CPU (host). Information is copied from CPU to GPU by utilizing cudaMemcpy keyword. A call using Kernel specifies the number of threads and blocks that are required by an application for the computation. A keyword _ global_ is prefixed with kernel keyword to distinguish from normal function. If keyword global is present in function declaration that function can be run on GPU. Only final Results are copied from the device to host in the same manner as information is copied from CPU to GPU, otherwise it will result in poor execution time.

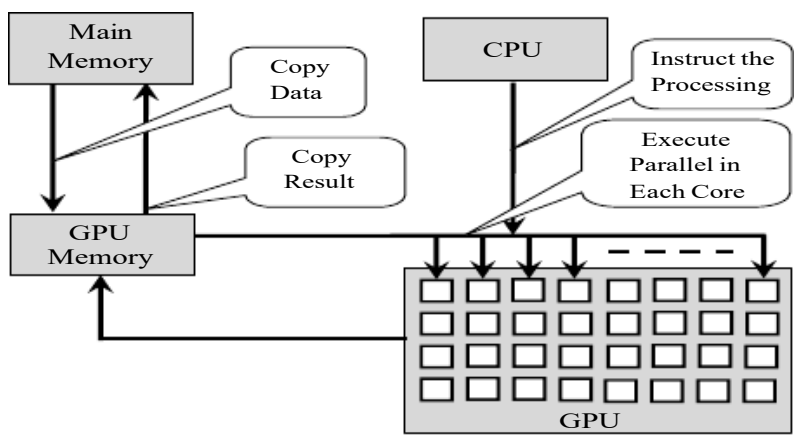

Figure 4 Data Flow CUDA Architecture

\section{MACKEY GLASS TIME SERIES}

Mackey Glass Time Series is a chaotic system described by Eq. (13)

$$
x(t)=a x(t-\tau) /\left(1+x^{10}(t-\tau)-b x(t)\right)
$$

where $\tau, a, b$ are parameters of the system. The result of equation is chaotic only when initial value of $a$ is set to $0.2, b$ is set to 0.1 and $\tau$ is s e t a s 17 .

By using the equation 13 the aim is to calculate futuristic value of time series at point next point $\mathrm{x}(\mathrm{t}+1)$ using past data given by points $\mathrm{x}(\mathrm{t}-18), \mathrm{x}(\mathrm{t}-12), \mathrm{x}(\mathrm{t}-6), \mathrm{x}(\mathrm{t})$. The process is modeled by Eq. (14)

$$
x(t)=f(x(t-18), x(t-12), x(t-6), x(t))
$$

In order to train an FLS, 700 data pairs were taken to evolve the rule base. Once rule base is evolved then it is used to predict the time series.

\section{IMPLEMENTATION OF TOOLBOX}

The figure for the toolbox is shown in Fig.5. This toolbox has options for both serial and parallel executions. If the system does not have a GPU it gives information to the user and uses CPU. Data file can be loaded using the toolbox. User can also change the color of toolbox. The toolbox can be used to detect the execution time of either T1 FLS or IT2 FLS. If GPU is available, the toolbox prints the message and executes the program in parallel. To change the color of toolbox button is provided. The button show graph on the toolbox is used to show graph between the actual output and the calculated output for an FLS. 


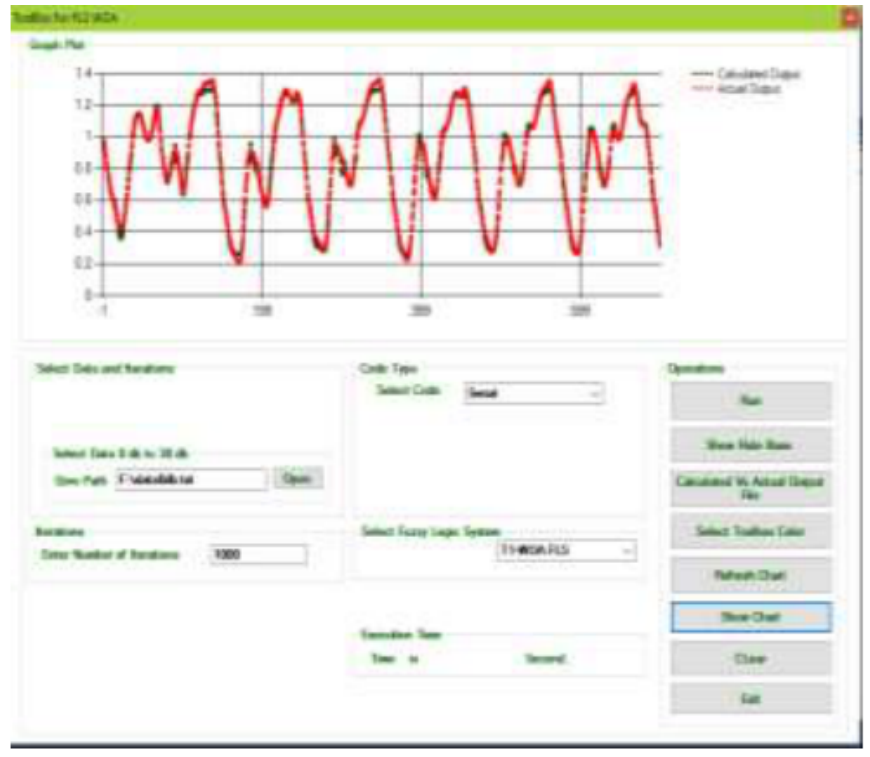

Figure 5 GUI for Toolbox

The button clear can be used to clear or reset the information filled by the user. The exit button is used to exit the program. The options serial and parallel execution gives a choice to the user to evolve the system in serial or parallel.

\subsection{Design of Toolbox}

The design followed for the toolbox is divided into phases. The first step of the design process is designing of the FLS. The FLS designing address the issue related to decision regarding number of fuzzy sets and type of fuzzy sets. The next stage of design is to decide the means and sigma matrices for the toolbox. Afterwards, programs for T1 FLS using WOA and IT2 FLS using WOA has been integrated in the toolbox.

The toolbox evolves the rule base of T1 FLS or IT2 FLS using WOA. The rule base evolved can be used for the prediction of any rule base system. The sample graph between actual values and calculated values is shown in the Fig. 6. From the figure it is observed that the output of the toolbox is acceptable.

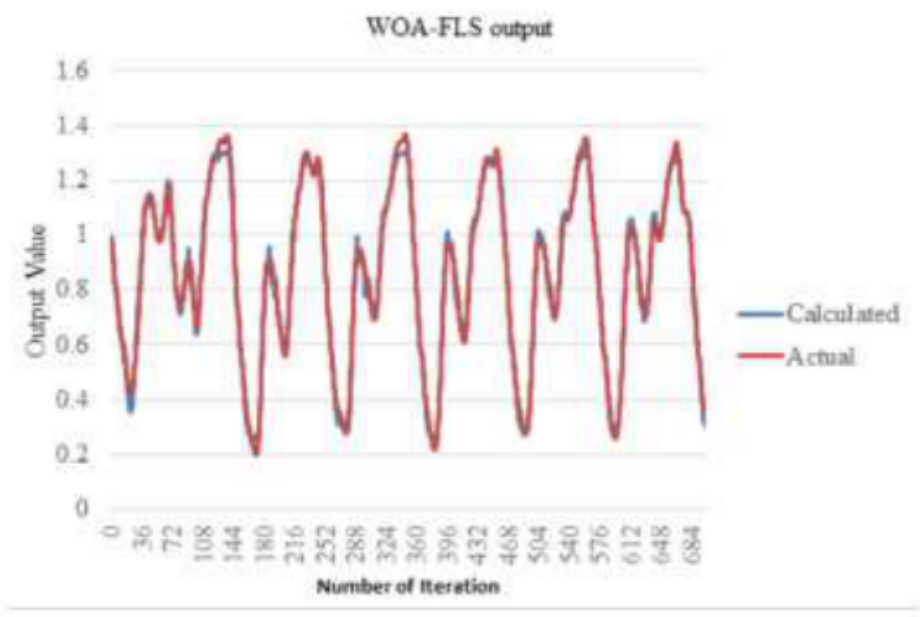

Figure 6 WO AFLS Output 


\section{RESULT AND DISCUSSION}

The results shown in this paper indicate that GPU begins to outperform CPU as the number of fuzzy rules increase. This experiment has been carried out using Intel i5, 4 GB RAM, and Windows 10. The GPU Nvidia Geforce GTX 940M is used for experimentation.

The toolbox is coded in Visual Studio C++ (2010), C $\backslash \#$ has been used for creation of Graphical User Interface for the toolbox. The results for comparison of serial execution are shown in the table 1. From the table it is clear that for zero db noise T1 FLS and IT2 FLS performance is same. However, as the noise level is increased IT2 starts giving better outputs as compared to T1 FLS.

Table 1 Comparison of RMSE of T1 WOA Serial and IT2 WOA Serial

\begin{tabular}{|c|c|c|}
\hline \multirow{2}{*}{$\begin{array}{c}\text { Noise } \\
\text { Level } \\
\text { in db }\end{array}$} & \multicolumn{2}{|c|}{ Comparison of RMSE of Serial Execution } \\
\cline { 2 - 3 } & T1- FLS & IT2-FLS \\
\hline 0 & 0.03 & 0.03 \\
\hline 10 & 0.39 & 0.25 \\
\hline 15 & 0.21 & 0.19 \\
\hline 20 & 0.23 & 0.19 \\
\hline 25 & 0.23 & 0.16 \\
\hline 30 & 0.24 & 0.20 \\
\hline
\end{tabular}

The above table show results for zero db, $10 \mathrm{db}, 15 \mathrm{db}, 20 \mathrm{db}, 25 \mathrm{db}, 30 \mathrm{db}$. As noise level zero both T1 FLS and IT2 FLs performs with same accuracy. As the noise level is increased to $10,15,20,25,30 \mathrm{db}$ respectively IT2 FLS starts performing better as compared to T1 FLS. Thus, it is observed that IT2 performs with better accuracy if signal is affected by noise.

The second simulation compares the results of parallel execution of T1 FLS and IT2 FLS integrated with WOA. The results for the same are tabulated in table 2. The results show that using GPU, the results of IT2 FLS using WOA is compared with that of IT2 FLS using CPU. The results of CPU and GPU are same. Hence, GPU can be used in place of CPU. The impact of using GPU results in same accuracy but with better execution time.

The results were repeated for thirty trials and average of results is carried out.

Table 2 Comparison of RMSE of IT2 WOA Serial and IT2 WOA Parallel

\begin{tabular}{|c|c|c|}
\hline \multirow{2}{*}{$\begin{array}{c}\text { Noise } \\
\text { Level } \\
\text { in db }\end{array}$} & \multicolumn{2}{|c|}{ Comparison of RMSE of Serial Execution } \\
\cline { 2 - 3 } & IT2-FLS Serial & IT2-FLS Parallel \\
\hline 0 & 0.03 & 0.03 \\
\hline 10 & 0.25 & 0.25 \\
\hline 15 & 0.19 & 0.19 \\
\hline 20 & 0.19 & 0.19 \\
\hline 25 & 0.16 & 0.16 \\
\hline 30 & 0.20 & 0.20 \\
\hline
\end{tabular}

The toolbox is used to calculate the execution time of the T1 FLS using WOA or IT2 FLS using WOA. 


\section{CONCLUSION}

In this paper, a toolbox is proposed that can be used to optimize the rule base in parallel. The toolbox uses Interval Type-2 Fuzzy Logic System, Whale Optimization Algorithm and Graphics Processing Unit (GPU). It gives variety of options to the user. The toolbox can be used to evolve any rule base using either T1 FLS or IT2 FLS. User can use either GPU is available or CPU. It prints execution time of the program. This will make the task of evolving the rule base of any fuzzy logic system easy and fast. FLS WOA Toolbox is design in such a way that user can pass parameter dynamically according to their need without interfacing with the code.

\section{REFERENCES}

[1] S. Mirjalili and A. Lewis, "The whale optimization algorithm", Advances in Engineering Software, vol 95, pp. 51-67, 2016.

[2] L. A. Zadeh, "Fuzzy Logic and Approximate Reasoning, Synthese", vol 30, Number 34, pp. 407-428, 1975.

[3] J. M. Mendel, Comparing the performance potentials of interval and general type-2 rulebased fuzzy systems in terms of sculpting the state space, IEEE Transactions on Fuzzy Systems, 2018.

[4] D. Luebke and M. Harris, General-purpose computation on graphics hardware, in Workshop, SIGGRAPH, 2004.

[5] J. D. Owens, D. Luebke, N. Govindaraju, M. Harris, J. Krüger, A.E. Lefohn, and T. J. Purcell, A survey of general-purpose computation on graphics hardware, in Computer graphics forum, vol 26, Number 1. Wiley Online Library, pp. 80-113. 2007

[6] I. Buck, T. Foley, D. Horn, J. Sugerman, K. Fatahalian, M. Houston, and P. Hanrahan, Brook for GPUS: stream computing on graphics hardware, in ACM transactions on graphics (TOG), Vol 23, Number 3. ACM, 2004, pp. 777-786.

[7] J. Sanders and E. Kandrot, DA by example: an introductio to general-purpose GPU programming. Addison-Wesley Professional, 2010.

[8] C. M. Fonseca and P. J. Fleming, "An overview of evolutionary Algorithms in multiobjective optimization," Evolutionary computation, vol. 3, no. 1, pp. 1-16, 1995.

[9] A. Biswas, K. Mishra, S. Tiwari, and A. Misra, "Physics-inspired optimization algorithms: a survey," Journal of Optimization, vol. 2013, 2013.

[10] R. S. Parpinelli and H. S. Lopes, "New inspirations in swarm intelligence: a survey," International Journal of Bio-Inspired Computation, Vol. 3, no. 1, pp. 1-16, 2011.

[11] J. H. Holland and J. S. Reitman, "Cognitive systems based on adaptive algorithms," ACM Sigart Bulletin, no. 63, pp. 49-49, 1977.

[12] I. BoussaïD, J. Lepagnot, and P. Siarry, "A survey on optimization metaheuristics," Information Sciences, vol. 237, pp. 82-117, 2013.

[13] S. Kirkpatrick, C. D. Gelatt, and M. P. Vecchi, "Optimization by simulated annealing," science, vol. 220, no. 4598, pp. 671-680, 1983.

[14] E. Rashedi, H. Nezamabadi-Pour, and S. Saryazdi, "GSA: a gravitational search algorithm," Information sciences, vol. 179, no. 13, pp. 2232-2248, 2009.

[15] S. Saremi, S. Mirjalili, and A. Lewis, "Grasshopper optimisation algorithm: Theory and application," Advances in Engineering Software, vol.105, pp. 30-47, 2017.

[16] L. Lin and M. Gen, "Auto-tuning strategy for evolutionary algorithms: balancing between exploration and exploitation," Soft Computing, vol. 13, no. 2, pp. 157-168, 2009. 
[17] E. Alba and B. Dorronsoro, "The exploration/exploitation tradeoff in dynamic cellular genetic algorithms," IEEE Transactions on Evolutionary Computation, vol. 9, no. 2, pp. 126-142, 2005.

[18] O. Olorunda and A. P. Engelbrecht, "Measuring exploration/exploitation in particle swarms using swarm diversity," in Evolutionary Computation,2008. CEC 2008. (IEEE World Congress on Computational Intelligence). IEEE Congress on. IEEE, pp. 1128-1134, 2008

[19] R. T. Marler and J. S. Arora, "Survey of multi-objective optimization methods for engineering," Structural and multidisciplinary optimization, vol. 26, no. 6, pp. 369-395, 2004.

[20] J. C. Spall, Introduction to stochastic search and optimization: estimation, simulation, and control, John Wiley \& Sons, vol. 65. 2005.

[21] X.-S. Yang, Nature-inspired metaheuristic algorithms, Luniver press, 2010.

[22] K. Deb, Multi-objective optimization using evolutionary algorithms. John Wiley \& Sons, vol. 16, 2001.

[23] S. Mirjalili and A. Lewis, "S-shaped versus v-shaped transfer functions for binary particle swarm optimization," Swarm and Evolutionary Computation, vol. 9, pp. 1-14, 2013.

[24] M. Dorigo, M. Birattari, and T. Stutzle, "Ant colony optimization," IEEE computational intelligence magazine, vol. 1, no. 4, pp. 28-39, 2006.

[25] R. Storn and K. Price, "Differential evolution-a simple and efficient heuristic for global optimization over continuous spaces," Journal of global optimization, vol. 11, no. 4, pp. 341-359, 1997.

[26] S. Mirjalili, "Sca: a sine cosine algorithm for solving optimization problems," KnowledgeBased Systems, vol. 96, pp. 120-133, 2016.

[27] A. Borovykh, S. Bohte, and C. W. Oosterlee, "Conditional time series forecasting with convolutional neural networks", arXiv preprint arXiv:1703.04691, 2017.

[28] U. Kose and A. Arslan, "Forecasting chaotic time series via anfis supported by vortex optimization algorithm: Applications on electroencephalogram time series," Arabian Journal for Science and Engineering, vol. 42, no. 8, pp. 3103-3114, 2017.

[29] N. Tak, A. A. Evren, M. Tez, and E. Egrioglu, "Recurrent type-1 fuzzy functions approach for time series forecasting," Applied Intelligence, vol. 48, no. 1, pp. 68-77, 2018.

[30] K. N. Murthy, R. Saravana, and K. V. Kumar, "Modeling and forecasting rainfall patterns of southwest monsoons in north-east india as a sarima process," Meteorology and Atmospheric Physics, vol. 130, no. 1, pp. 99-106, 2018.

[31] J. P. González, A. M. San Roque, and E. A. Pérez, "Forecasting functional time series with a new hilbertian armax model: Application to electricity price forecasting," IEEE Transactions on Power Systems, vol. 33, no. 1, pp. 545-556, 2018.

[32] Q. Liang and J. M. Mendel, "Interval type-2 fuzzy logic systems: theory and design," Fuzzy Systems, IEEE Transactions on, vol. 8, no. 5, pp.535-550, 2000.

[33] L. A. Zadeh, "Fuzzy Sets," Information and control, vol. 8, no. 3, pp.338-353, 1965.

[34] J. M. Mendel, "Fuzzy Logic Systems for Engineering: A Tutorial", Proceedings of the IEEE, vol. 83, no. 3, pp. 345-377, 1995.

[35] G. C. Mouzouris and J. M. Mendel, "Nonsingleton fuzzy logic systems: theory and application,” Fuzzy Systems, IEEE Transactions on, vol. 5, no. 1, pp. 56-71, 1997.

[36] N. N. Karnik and J. M. Mendel, "Applications of type-2 fuzzy logic systems to forecasting of time-series," Information Sciences, vol. 120, no. 1, pp. 89-111, 1999.

[37] J. M. Mendel, "Uncertainty, fuzzy logic, and signal processing," Signal Processing, vol. 80, no. 6, pp. 913-933, 2000. 
[38] M. Khosla, R. K. Sarin, M. Uddin, A. Khosla, and S. Singh, "Realizing interval type-2 fuzzy systems with type-1 fuzzy systems," Book Chapter for Book titled Cross Disciplinary Applications of Artificial Intelligence and Pattern Recognition: Advancing Technologies, IGI Global, USA. (Accepted for Publication), 2011.

[39] N. Harvey, R. Luke, J. M. Keller, and D. Anderson, "Speedup of fuzzy logic through stream processing on graphics processing units," in Evolutionary Computation, 2008. CEC 2008. (IEEE World Congress on Computational Intelligence). IEEE Congress on. IEEE, 2008, pp.3809-3815. 2008.

[40] D. Anderson and S. Coupland, "Parallelisation of fuzzy inference on a graphics processor unit using the compute unified device architecture," in Proceedings of the UK Workshop on Computational Intelligence (UKCI'08), pp. 1-6, 2008.

[41] L. T. Ngo, D. D. Nguyen, C. M. Luong et al, "Speedup of interval type 2 fuzzy logic systems based on GPU for robot navigation", Advances in Fuzzy Systems, vol. 2012, p. 4, 2012. 